\section{Gute Erfahrung mit CitraFleet ${ }^{\circledR}$ in der Endoskopievorbereitung}

CitraFleet $^{\circledR}$ ist ein apothekenpflichtiges Abführmittel zur Darmreinigung und kommt zum Einsatz bei diagnostischen Untersuchungen, die nur bei einem gut gereinigten Darm sinnvoll durchgeführt werden können, z.B. eine Koloskopie oder Röntgenuntersuchung. Es handelt sich um ein Kombinationspräparat aus den beiden Wirkstoffen Natriumpicosulfat und Magnesiumcitrat. Die mittels Magnesiumcitrat osmotisch induzierte Darmspülung wird durch die Motilitätssteigerung mit Picosulfat unterstützt. Die Kombination aus Natriumpicosulfat und Magnesiumcitrat findet in Großbritannien schon seit Jahren als Standardpräparat in der Endoskopievorbereitung Verwendung und wird seit Oktober 2008 durch die Eisai GmbH auch in Deutschland vertrieben.

\section{Gute Erfahrung durch hohe Wirksamkeit}

CitraFleet ${ }^{\circledR}$ hat sich in den prospektiven klinischen Studien als hochwirksam erwiesen. In der Gesamtübersicht der Studien liegt der Anteil der Patienten mit adäquatem Reinigungsergebnis nach Einnahme von CitraFleet ${ }^{\circledR}$ bei ca. $80 \%[1,2]$. In großen beobachtenden Studien zur Koloskopievorbereitung mit üblichen Darmvorbereitungsregimen liegt diese Quote bei durchschnittlich 75\% [3, 4]. Die verfügbaren Daten zur Reinigungsqualität bestätigen, dass CitraFleet ${ }^{\circledR}$ die Anforderungen der klinischen Praxis voll erfüllt.

\section{Hohe Sicherheit und Verträglichkeit}

Ebenfalls konnte in mehreren prospektiven Studien, in denen die Verträglichkeit von
Viszeralmedizin 25 | 2 | 09

CitraFleet ${ }^{\circledR}$ mit konventionellen Darmvorbereitungsregimen (Natriumphosphat, PEGLösungen) verglichen wurde, ein deutlicher Vorteil für CitraFleet ${ }^{\circledR}$ gezeigt werden. Insbesondere traten signifikant weniger gastrointestinale Beschwerden auf [5]. Die Verträglichkeit ist bei älteren Patienten ebenso gut wie bei jüngeren Erwachsenen [6]. CitraFleet ${ }^{\circledR}$ hat keinen erheblichen Einfluss auf den Wasserhaushalt und damit assoziierte Kreislaufparameter. Ausgeprägte Elektrolytstörungen und Dehydratation sind bei ausreichender Flüssigkeitssubstitution selten [7]. Da CitraFleet ${ }^{\circledR}$ kein Phosphat enthält, ist das Risiko für eine Phosphatnephropathie ausgeschlossen.

\section{Verbesserte Patientencompliance}

Hohes Einnahmevolumen, schlechter Geschmack und gastrointestinale Nebenwirkungen werden von vielen Patienten an den herkömmlichen Darmreinigungsregimen kritisiert. Hier bietet CitraFleet ${ }^{\circledR}$ einen wesentlichen Benefit, denn das Wirkstoff-Einnahmevolumen ist mit $2 \times 150 \mathrm{ml}$ im Abstand von 6-8 Stunden um das 6-13-fache geringer als beispielsweise bei PEG-Lösungen. Weitere Flüssigkeit wird durch die Einnahme von klaren Getränken substituiert. Der Geschmack von CitraFleet ${ }^{\circledR}$ wurde von den Teilnehmern mehrerer Studien gegenüber den Vergleichssubstanzen als signifikant angenehmer beurteilt $[8,9]$. Entsprechend ist die Akzeptanz der Patienten für die Darmvorbereitung mit CitraFleet ${ }^{\circledR}$ signifikant höher als für andere Regime, wodurch sich auch die Compliance deutlich verbessert: Wesentlich mehr Patienten nahmen die Darmvorbereitungslösung vollständig ein und wesentlich weniger Patienten gaben Schwierigkeiten bei der Einnahme an.

\section{Fazit}

Mit CitraFleet ${ }^{\circledR}$ steht ein niedrigvolumiges Darmreinigungspräparat zur Verfügung, das prospektiv gut dokumentiert ist und sich durch fehlendes Nephropathierisiko sowie gute Verträglichkeit in der klinischen Praxis auszeichnet. Geringes Wirkstoff-Einnahmevolumen kombiniert mit frei wählbarer Flüssigkeit, der angenehme Geschmack und reduzierte gastrointestinale Nebenwirkungen steigern die Akzeptanz und die Compliance der Patienten. Letzteres ist von großer Bedeutung für die Koloskopie, denn nur eine vollständige Reinigung gewährleistet eine aussagekräftige Untersuchung des Darms.

\section{Literatur}

Renault AJ.: Colorectal Dis 2007;10:503-5.

2 Navarro A.: 16th United European Gastroenterology Week, \#187 plus poster. 2008;18-22.

3 Harewood GC.: Gastrointest Endosc 2003;58:76-9.

4 Froehlich F.: Gastrointest Endosc 2005;61:378-84.

5 Hamilton D.: Br J Clin Pract 1996;50:73-59.

6 Heymann TD: BMJ 1996:313:727-8.

7 Barkun A.: Can J Gastroenterol 2006;20:699-710.

8 Hawkins S.: Australas Radiol 1995;68:128-9.

9 Worthington J.: Curr Med Res Opin 2008;24(2):481-8.

Weitere Informationen bei

Eisai $\mathrm{GmbH}$

Thomas Römmelt

Produktmanager GE Gastroenterologie Lyoner Str. 36, 60528 Frankfurt/Main Tel. +49 69 66585-89, Fax -25 thomas_roemmelt@eisai.net

\title{
Ticker+++ Ticker+++ Ticker+++ Ticker+++ Ticker+++ Ticker+++ Ticker+++
}

ERBE Elektromedizin. HybridKnife deckt als Multifunktionssonde alle Arbeitsschritte bei der endoskopischen Submukosa Dissektion (ESD) ab. Von der Markierung und Elevation der Mukosa bis hin zur Inzision/Dissektion der Läsion und Nachkoagulation des Resektionsbetts ist kein Instrumentenwechsel bei diesem minimalinvasiven Eingriff erforderlich.

Weitere Informationen be

ERBE Elektromedizin $\mathrm{GmbH}$

Thomas Hämmerle

Tel. +49 7071 755-138
Ethicon Endo-Surgery. In einem neu eingerichteten High-Tech Labor im European Surgical Institute (ESI) trainieren Chirurgen jetzt komplexe minimalinvasive Eingriffe realitätstreu. Die Schulung ist weltweit einzigartig: An 12 Simulationscomputern üben die Chirurgen komplizierte Eingriffe wie Darmoperationen am «virtuellen Patienten». Ziel ist die Qualitätsverbesserung bei höherer Patientensicherheit.

Weitere Informationen bei

medical relations $\mathrm{GmbH}$

Petra Sittauer

Tel. +49 2173 9769-0
Bayer Vital. Grundlage der Zulassung von Nexavar $^{\circledR}$ (Sorafenib) für die Behandlung von Patienten mit hepatozellulärem Karzinom waren 2 große Phase-II-Studien. Bei der 44. Jahrestagung der European Association for Study of the Liver im April 2009 präsentierten mehrere international renommierte HCC-Experten die neuesten Daten, die die Wirksamkeit und Sicherheit von Sorafenib bei verschiedenen Patientenpopulationen bestätigen.

Weitere Informationen be

Bayer Vital GmbH

Dr. Herbert Schäfer

Tel. +49 2143051109 